\title{
Seismic preprocessing and amplitude cross-calibration for a time-lapse amplitude study on seismic data from the Oseberg reservoir
}

\author{
E. Stucchi, * A. Mazzotti† and S. Ciuffi $\ddagger$ \\ Department of Earth Sciences/Geophysics, University of Milan, Via Cicognara 7, 20129 Milan, Italy
}

Received May 2004, revision accepted October 2004

\begin{abstract}
The cross-calibration of different vintage data is an important prerequisite in attempting to determine the time-lapse seismic effects induced by hydrocarbon production in a reservoir. This paper reports the preprocessing and cross-calibration procedures adopted to modify the data of four seismic vintages (1982, 1989, 1992 and 1999) from the Oseberg field in the North Sea, for optimal conditions for a time-lapse seismic amplitude analysis. The final results, in terms of time-lapse variations, of acoustic impedance and of amplitude-versus-offset, are illustrated for selected data sets. The application of preprocessing to each individual vintage data set reduces the effects of the different acquisition and noise conditions, and leads to consistency in the amplitude response of the four vintages. This consistency facilitates the final amplitude cross-calibration that is carried out using, as reference, the Cretaceous horizon reflections above the Brent reservoir. Such cross-calibration can be considered as vintage-consistent residual amplitude correction.

Acoustic impedance sections, intercept and gradient amplitude-versus-offset attributes and coherent amplitude-versus-offset estimates are computed on the final cross-calibrated data. The results, shown for three spatially coincident 2D lines selected from the 1982, 1989 and 1999 data sets, clearly indicate gas-cap expansion resulting from oil production. Such expansion is manifested as a decrease in acoustic impedance and a modification of the amplitude-versus-offset trends in the apical part of the reservoir.
\end{abstract}

\section{INTRODUCTION}

Since the early experiments of repeated seismic surveys to check fire-flood effects on a hydrocarbon reservoir (Greaves and Fulp 1987), time-lapse seismic surveys have been increasingly employed to monitor the evolution of producing reservoirs. This innovative methodology is referred to extensively in the literature, and its development and applications are among the main topics of international scientific meetings (Lumley, Behrens and Wang 1997; De Waal et al.2001; Parker,

*E-mail: eusebio.stucchi@unimi.it

$\dagger$ Now at: Department of Earth Sciences, University of Pisa, Via S. Maria 53, 56126 Pisa, Italy.

$\ddagger$ Now at: Enel Green Power, Via A. Pisano 120, 56122 Pisa, Italy.
Bertelli and Dromgoole 2003). It should be noted, however, that the final applicability of time-lapse seismic methods to reservoir production monitoring depends on factors (Lumley 2001) such as:

1 suitable petrophysical and production conditions (see, e.g., Wang 1997) that may or may not produce time-lapse, production-dependent, seismic signatures above the data noise level;

2 acquisition procedures that should ensure repeatability of the seismic measurements, and that maintain source bandwidth, offset and azimuth ranges, bin coverage and array responses;

3 processing and calibration procedures that remove the different acquisition footprints, reduce the noise and retrieve the required time-lapse signatures. 
The work presented here was carried out, in collaboration with industrial partners, within the framework of a research project in which various seismic methodologies, such as true-amplitude prestack depth migration, reflection tomography, signal-amplitude analysis and inversion, were investigated to verify their applicability for time-lapse seismic studies (Bush et al. 2000; Mazzotti, Stucchi and Ciuffi 2000; Rowbotham et al. 2001; Stucchi, Mazzotti and Terenghi 2001; Vesnaver et al. 2001, 2003; Hicks and Williamson 2002).

The 4D seismic data pertain to the Oseberg field in the Norwegian North Sea (Johnsen, Rutledal and Nilsen 1995). The reservoir rocks are within the Brent Group where porosity ranges from $20 \%$ to $27 \%$, and thickness from $40 \mathrm{~m}$ to 200 $\mathrm{m}$. The Oseberg Formation is the main reservoir while the overlying Cretaceous limestone and shale forms the seal. Oil production from the Brent started in 1988. Gas injection and oil production were tuned to maintain stability in the reservoir pressure and the gas-front movement. A previous feasibility study on synthetic seismic data indicated that variations in the saturation of the reservoir due to production would give rise to subtle but noticeable effects on seismic response, particularly amplitude changes. Previous studies on 4D seismic monitoring from 1989 to 1992 can be found in Johnstad, Seymour and Smith (1995).

Our efforts were focused on finding discernible amplitude indicators of saturation changes in the reservoir during production. Thus, the preprocessing of each single-vintage data set, the two different types of inter-vintage amplitude calibration and the extraction of the time-lapse amplitude variations in terms of acoustic impedance and amplitude-versus-offset attributes are reported. In particular, we show the results pertaining to single 2D lines extracted from four 3D streamer surveys acquired in 1982, 1989, 1992 and 1999, situated in approximately coincident spatial locations (Fig. 1).

Whereas a thorough cross-calibration requires a space- and time-variant matching of both the amplitude and phase characteristics of the different vintage signals (Harris and Henry 1998; Rickett and Lumley 2001), our approach considers only signal amplitudes. Spectral matching was carried out only on the post-stack data, before the computation of the acoustic impedance.

In evaluating the time-lapse amplitude signatures we followed a target-orientated approach that is focused on the

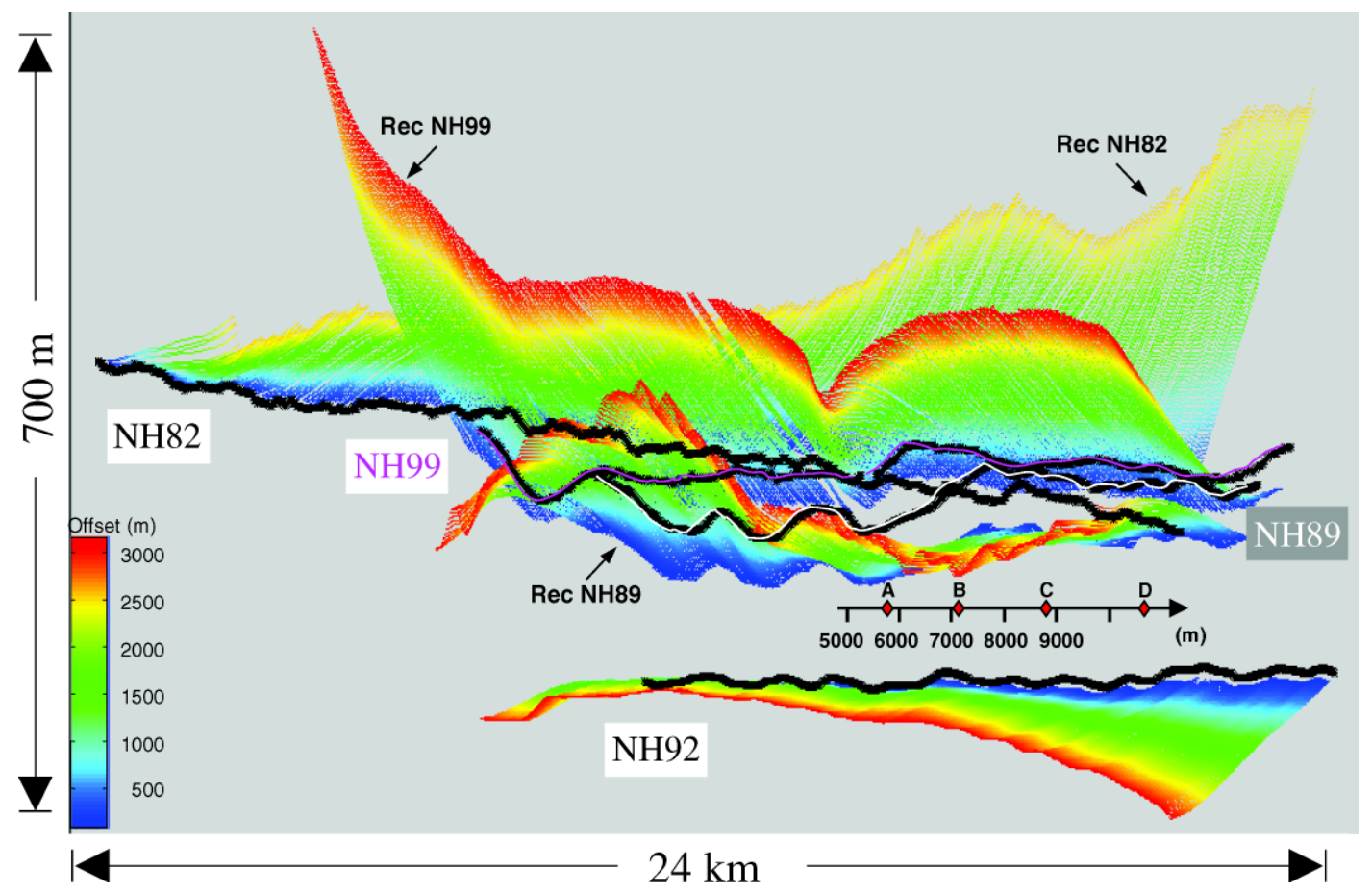

Figure 1 Map of the seismic data used throughout this work. The black dots represent the shot positions, while the receiver positions are plotted with different colours according to the source-to-receiver offset. Note the different scales of the horizontal and vertical axes. The $x$-axis with coordinates plotted inside the map and the locations A, B, C and D are used as references further on. 
response from the reservoir layer and from a shallower layer (Top Cretaceous) as reference. The exercise was carried out 'blind', that is, with no a priori information on either fluid movement or reservoir production data. Thus, what we discuss here pertains to a purely seismic perspective. There is no discussion in the present paper of the added value of applying true-amplitude prestack depth migration (Tura, Hanitzsch and Calandra 1998), which requires an accurate knowledge of the velocity field.

\section{DESCRIPTION OF THE AVAILABLE DATA}

The map (Fig. 1, not to scale) shows the location of the 2D lines available for this study in the Alpha block of the Oseberg field. The shot positions are represented by black dots, while the receiver positions are plotted in colour according to the offset. Note the different feathering of the streamer during field acquisition. The lines extracted from the 1982, 1989 and 1999 vintage data are practically coincident, while the 1992 line is shifted about $250 \mathrm{~m}$ to the south. For this reason, when assessing the time-lapse changes in the reservoir, no account was taken of the 1992 line.
The field acquisition parameters of the four vintages varied significantly, having different minimum and maximum available offsets, source and receiver arrays, receiver spacing, coverage and acquisition technology (single/multiple sources and single/multiple streamers). Furthermore, all the vintage data are affected by major noise problems: water-bed and peg-leg multiples, diffraction tails from shallower objects, and coherent noise intersecting the target reflectors. The close-up (Fig. 2) of the stack section from the 1999 vintage is centred on the apical part of the Brent reservoir, close to the fault separating the Alpha and Gamma blocks (Johnsen et al. 1995). The data shown are the end result of the single-vintage processing that will be discussed later. For reference purposes, Fig. 2 shows the relevant reflectors, a time window that includes the reflections from the Cretaceous layer and, along the line, four positions A, B, C, D.

The sonic and density logs from a nearby well are shown in Fig. 3. Note the marked increase in the P-wave velocity of the Cretaceous layer, and the significant decrease in the density and $V_{\mathrm{P}} / V_{\mathrm{S}}$ ratio of the Brent reservoir. These borehole data were used to perform post-stack wavelet processing to increase resolution at the target level, before the computation of

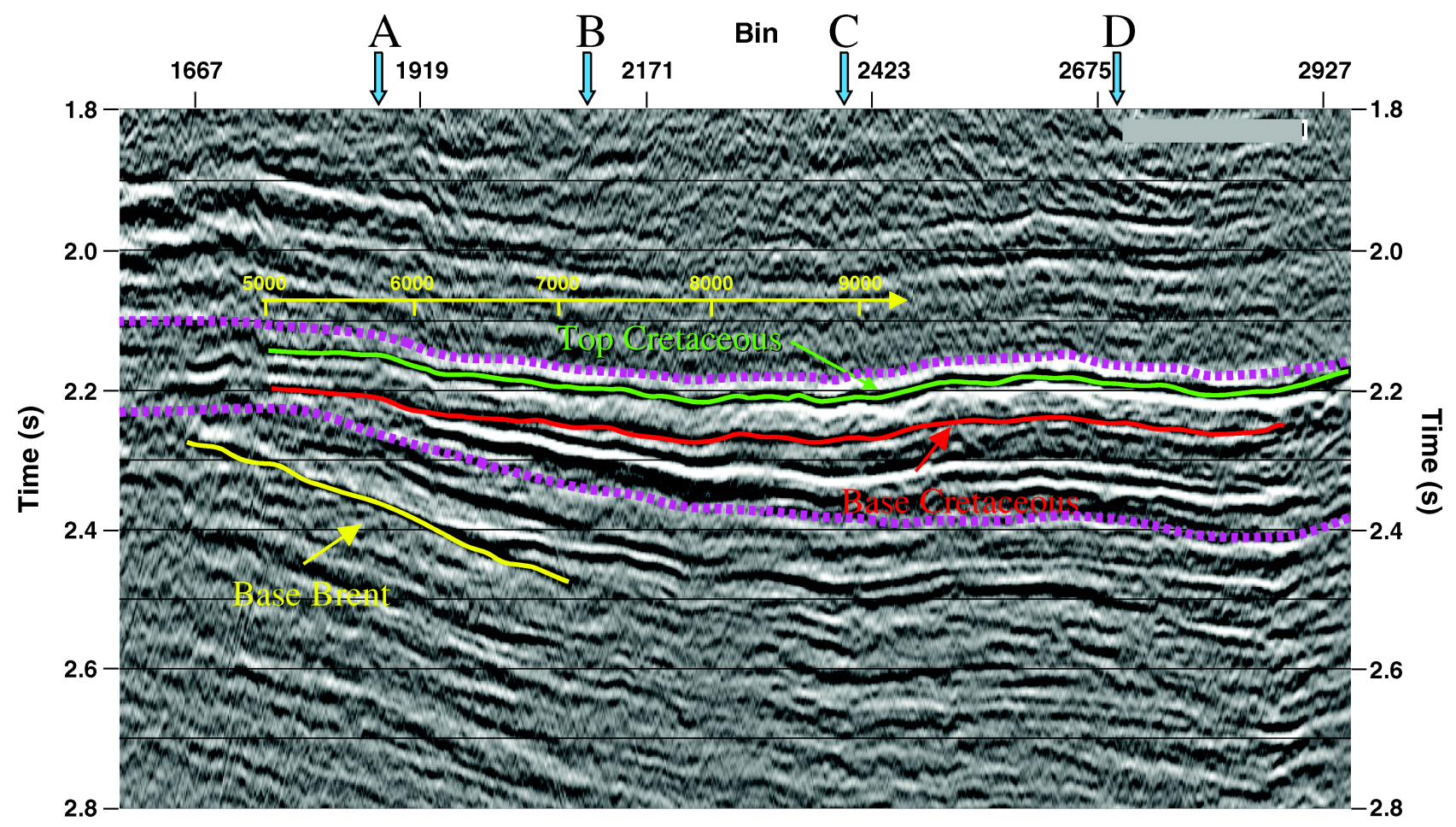

Figure 2 Stack section of the 1999 data at the end of the single-vintage processing. Relevant target reflectors are indicated. The pink dots delimit the time gate of data used for the data cross-calibration procedure. The yellow $x$-axis overprinted on the stack section gives the bearings with respect to the map of Fig. 1; the locations A, B, C and D are used as references further on. 


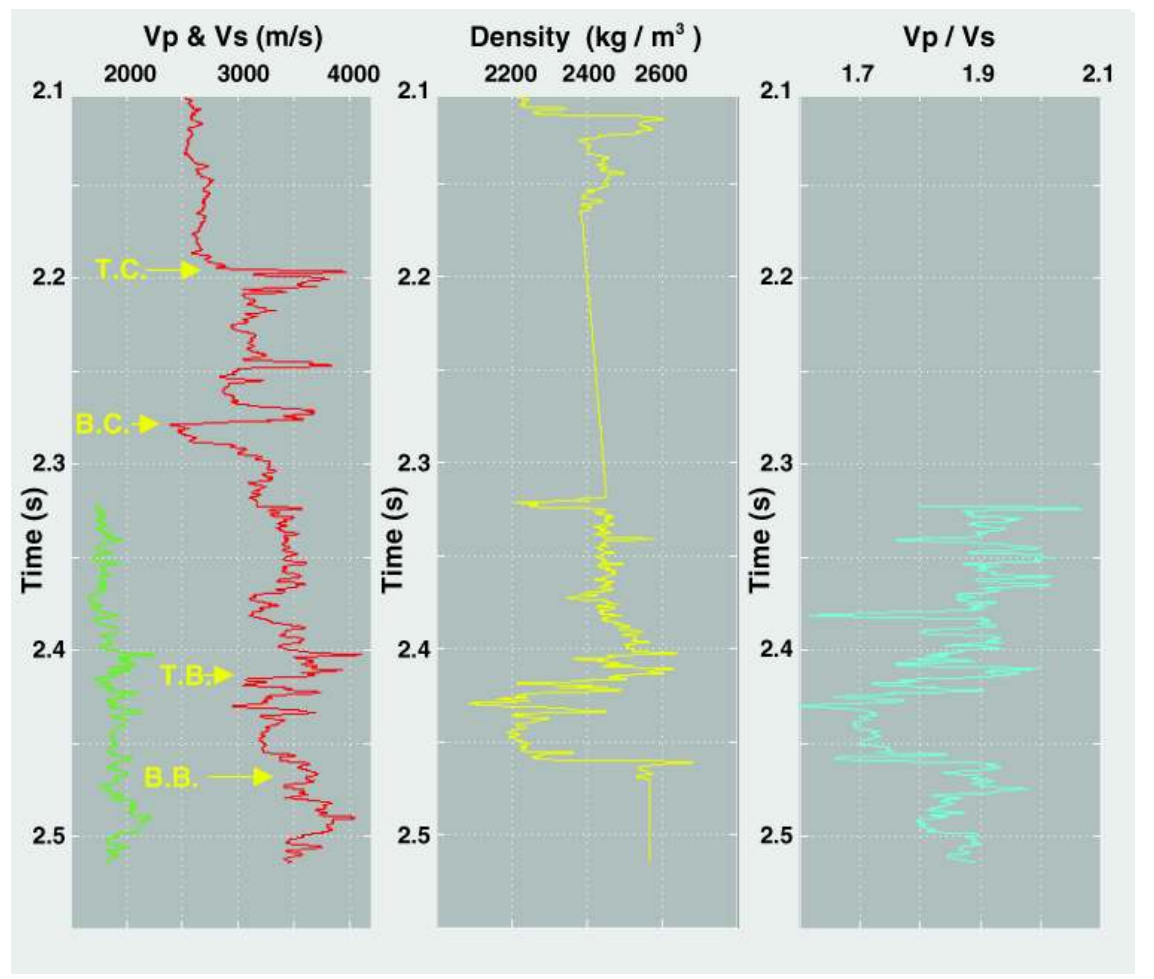

Figure 3 Borehole logs from a well close to location $\mathrm{C}$ in Fig. 2. T.C., Top Cretaceous; B.C., Base Cretaceous; T.B., Top Brent; B.B., Base Brent.

the seismic acoustic impedance (Terenghi and Mazzotti 2002). Prestack wavelet processing was attempted in order to adjust the wavelets of the different vintages to a common shape, but this met with little success due to the significant noise contamination in the prestack data.

\section{SINGLE-VINTAGE PROCESSING}

The processing sequence applied to each single-vintage data set is briefly described below. First we applied true-amplitude processing, the amplitude recovery depending on the specific features of each vintage. This was followed by surface-consistent residual-amplitude compensation and surface-consistent multiple attenuation. Table 1 shows the most significant steps of the processing applied to the data.

In all the vintages, attenuation due to source and receiver arrays was corrected for, although, given their limited dimensions, their effect was not particularly critical at the times of the target reflectors. However, a limited differential effect among the vintages does exist. Let us look, for example, at the 1989 and 1992 data at the Base Brent reflector time of $2.5 \mathrm{~s}$ : the source array correction for $50 \mathrm{~Hz}$ frequency at an offset of $3000 \mathrm{~m}$ increases the amplitudes, due to the different configuration of the airguns, i.e. $1.7 \%$ and $9.2 \%$. Instead geometrical spreading has a strong impact on the Brent reflector amplitudes, but, since we used a common-velocity field for
Table 1 Main steps of the single-vintage processing

Bad trace and spike editing

Band-pass filtering

Source and receiver array compensation

Offset-dependent geometrical spreading (using a common velocity field)

Surface-consistent amplitude corrections

Surface-consistent predictive deconvolution

Gapped predictive deconvolution

the computation of the spreading factor, there was no differential effect among the different vintages.

An important step in amplitude compensation is surfaceconsistent amplitude correction (Taner and Koehler 1981). This is because it leads to a more homogeneous amplitude status for the various vintage amplitudes. Such correction is generally effective in compensating for source and receiver efficiency variations, and for other near-surface effects. The rms amplitude value in a $1 \mathrm{~s}$ to $3 \mathrm{~s}$ time gate is computed for each trace after an accurate muting to exclude first arrivals. The surface-consistent decomposition consists of separating the observed trace amplitudes into shot, receiver and bin components. The subsequent corrections are computed and applied to adjust the shot and the receiver components to constant values. As an example, Fig. 4 shows the shot component, 
computed for a portion (nine lines) of the entire 3D data set from the 1989 data, before and after the application of these corrections. It can be seen that the source term is not constant along the profile, and is likely to introduce amplitude distortion into the data. Effects of similar magnitude but located in different spatial positions are present on the data of the other vintages. Thus, if these effects are not compensated for, they can leave incorrect time-lapse amplitude signatures in the data.
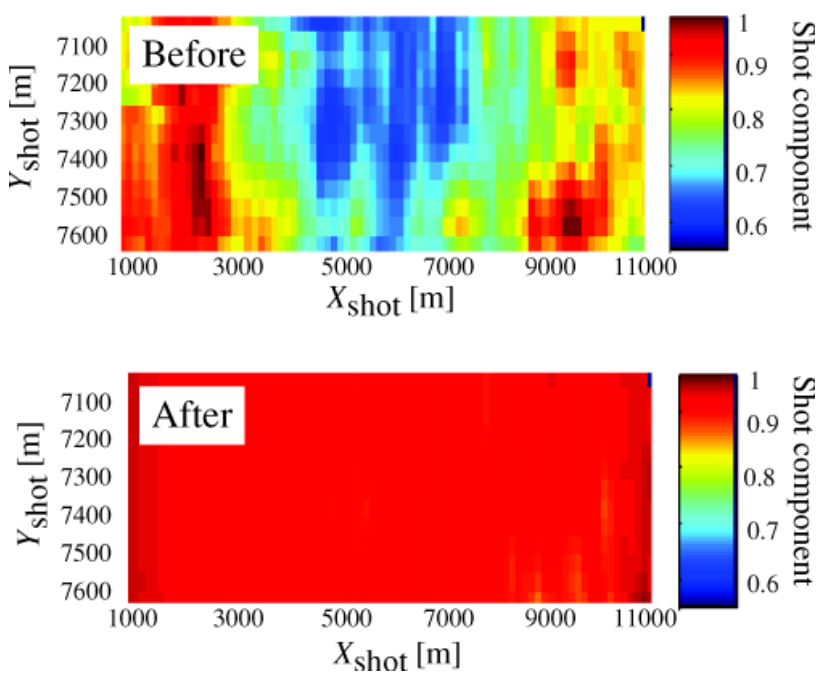

Figure 4 The upper frame shows the shot amplitude component before surface-consistent amplitude corrections. The lower frame shows the same component after surface-consistent amplitude correction. In both cases, geometrical spreading and array directivity corrections are present. Both images refer to 1989 data and X, Y represent the shot coordinates.
An interesting feature that we found in the amplitude responses while analysing the prestack data was the presence of some anomalous high amplitudes, located approximately at the same spatial coordinates in all four vintage data sets. Initially, we thought they might be due to the interference of coherent noise, such as diffractions from drilling platforms or from other sea-bottom equipment. A more detailed study employing traveltime tomography as well as amplitude analysis revealed that such high amplitudes are caused by low-velocity mud diapirs (about $200 \mathrm{~m}$ thick) located at shallower depths, which produce significant focusing effects. As an example, Fig. 5 shows a bin gather where amplitude focusing and traveltime delays are evident at the central offsets.

Multiple reflections, both water-bottom and interbed, severely contaminate the data and constitute a major problem. After several tests, we decided to apply surface-consistent predictive deconvolution (Morley and Claerbout 1983; Levin 1989). In the decomposition we separated four components: shot, receiver, offset and bin. The terms shot, receiver and offset were used to define the operators. For each single vintage, the prediction distances and filter lengths were decided on the basis of the autocorrelation of common-offset traces. To tackle the different periodicity at the different offsets, and also the different order of the multiples, a second pass of predictive deconvolution was needed. The relevant parameters for each vintage are given in Table 2 . The first pass deals with the multiples that determine a peak around $0.06 \mathrm{~s}$ in the autocorrelation, while the second pass deals with the multiples which cause another relative maximum at about $0.120 \mathrm{~s}$ in the autocorrelation. The slight differences in the deconvolution parameters of the vintages depend mainly on the different

Figure 5 Close-up of a bin gather from the 1999 data set after surface-consistent compensation and normal-moveout correction. Amplitude anomalies and small traveltime delays clearly stand out in the area included in the red box. These effects are due to focusing produced by shallower mud diapirs.

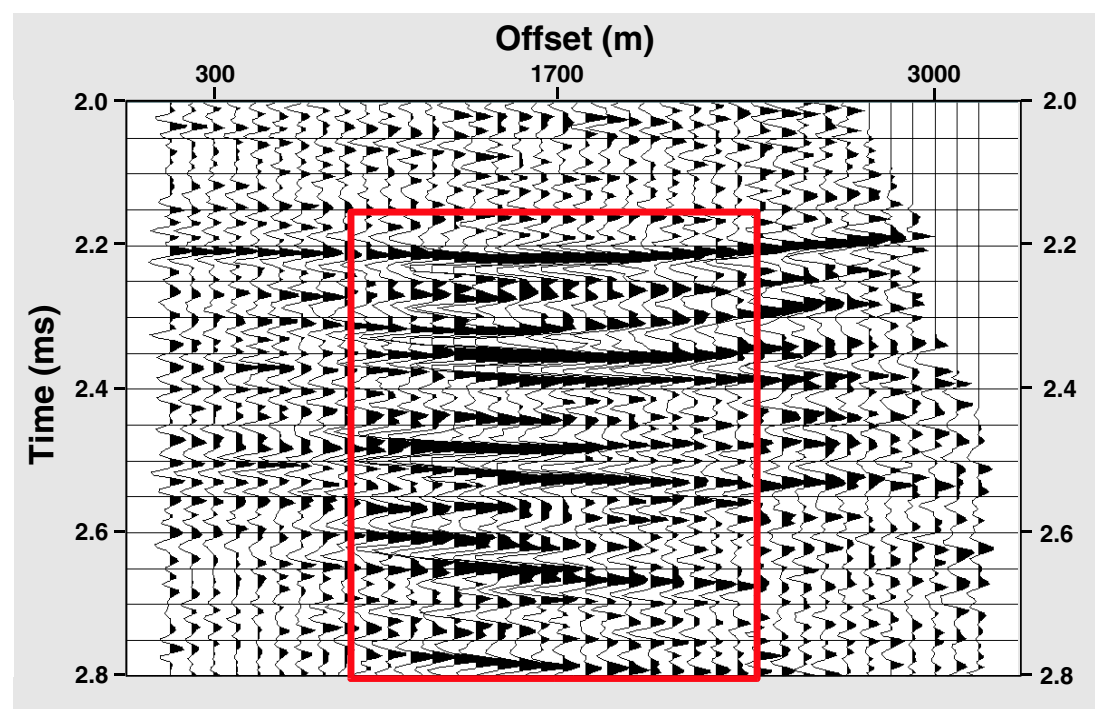


Table 2 Deconvolution parameters for the different vintage data

\begin{tabular}{|c|c|c|c|c|c|c|c|c|}
\hline \multirow[b]{2}{*}{ Deconvolution parameters } & \multicolumn{2}{|l|}{1982} & \multicolumn{2}{|l|}{1989} & \multicolumn{2}{|l|}{1992} & \multicolumn{2}{|l|}{1999} \\
\hline & 1 st pass & 2 nd pass & 1 st pass & 2nd pass & 1 st pass & 2nd pass & 1 st pass & 2 nd pass \\
\hline Temporal window & $0.5-3.0 \mathrm{~s}$ & $0.5-3.0 \mathrm{~s}$ & $0.5-3.0 \mathrm{~s}$ & $0.5-3.0 \mathrm{~s}$ & $0.5-3.0 \mathrm{~s}$ & $0.5-3.0 \mathrm{~s}$ & $0.5-3.0 \mathrm{~s}$ & $0.5-3.0 \mathrm{~s}$ \\
\hline Prediction distance & $0.018 \mathrm{~s}$ & $0.1 \mathrm{~s}$ & $0.014 \mathrm{~s}$ & $0.1 \mathrm{~s}$ & $0.016 \mathrm{~s}$ & $0.1 \mathrm{~s}$ & $0.016 \mathrm{~s}$ & $0.1 \mathrm{~s}$ \\
\hline Filter length & $0.13 \mathrm{~s}$ & $0.1 \mathrm{~s}$ & $0.17 \mathrm{~s}$ & $0.1 \mathrm{~s}$ & $0.18 \mathrm{~s}$ & $0.1 \mathrm{~s}$ & $0.18 \mathrm{~s}$ & $0.1 \mathrm{~s}$ \\
\hline
\end{tabular}

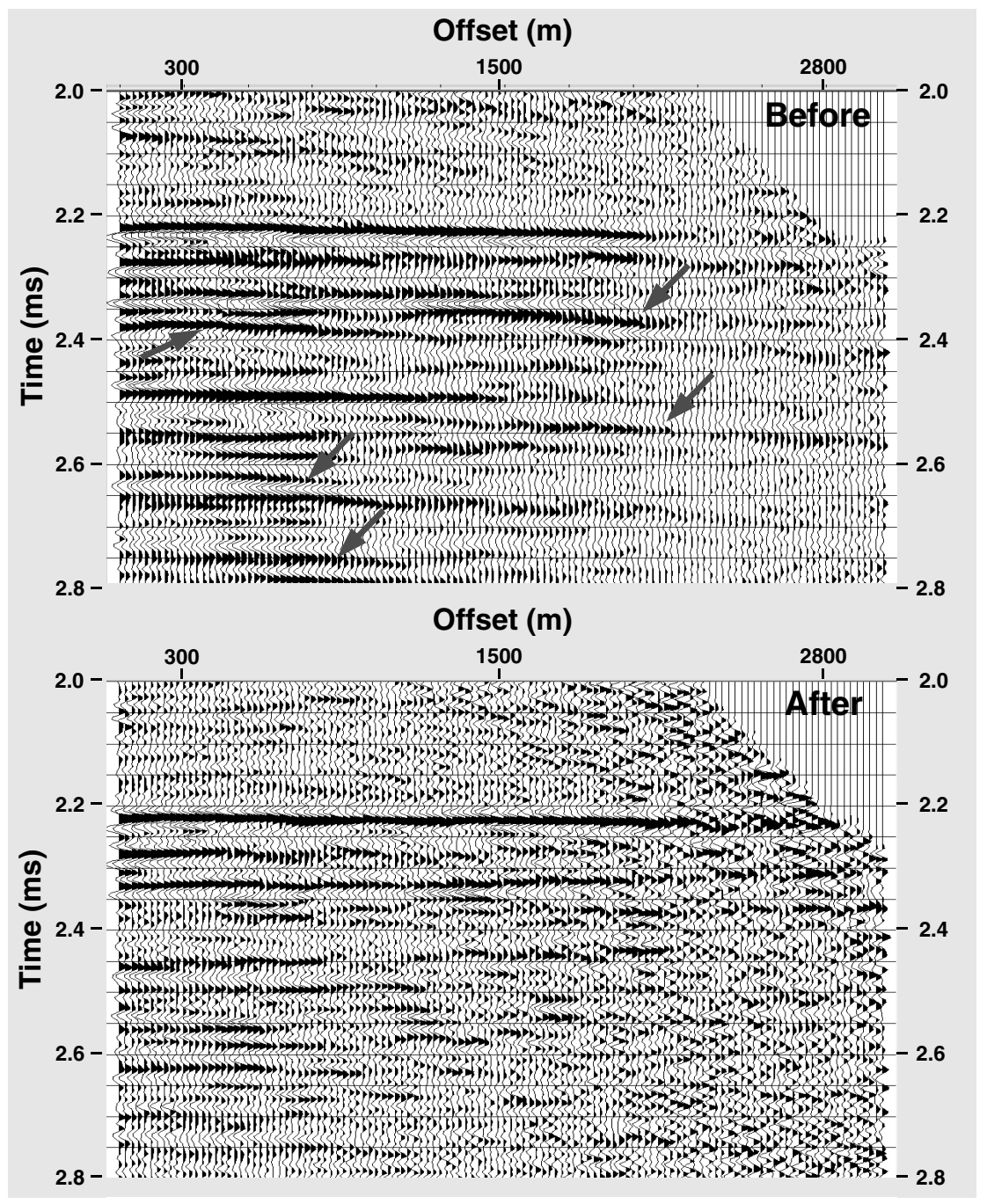

Figure 6 Bin gather from the 1989 vintage near location C in Fig. 2, before (top) and after (bottom) single-vintage processing. The arrows show multiple reflections removed by the deconvolution steps. Also note the recovery of amplitudes at higher offsets and times. The slight increase in noise is due to the combined effects of deconvolution and gain application.

wavelet bandwidths, with the 1989 data having the largest frequency band.

More sophisticated techniques of multiple removal, such as those based on wave-equation approaches (e.g. Verschuur and Berkhout 1997; Lokshtanov 2000; Spitz 2000), may be more effective in attenuating multiple energy, but our results can be considered adequate for the scope of our time-lapse amplitude analysis.
Figure 2 shows a portion of the stack section of the 1999 vintage at the end of the single-vintage processing. Figure 6 shows a bin gather from 1989 close to the location labelled $\mathrm{C}$ in Fig. 2, before and after the single-vintage processing. Amplitude restoration and the attenuation of multiples can be observed.

It can be seen that, despite the coherent noise intersecting the target reflections, there are no operations aimed at 
its removal in the sequence. We tried various kinds of multichannel filtering in the $t-x, f-k$ and $\tau-p$ domains, but were unable to remove the coherent noise satisfactorily without introducing artefacts and strong lateral mixing effects into the data amplitude and, in particular, into the amplitude-versusoffset response. Furthermore, different trace spacings among the vintages, which created different aliasing conditions, prevented a confident use of such techniques in a time-lapse sense. However, in many cases this kind of noise, which is primarily due to diffraction tails from shallower objects, is repetitive and thus does not preclude a time-lapse analysis of the target amplitudes. Also, to extract more reliable, noise-free amplitude-versus-offset responses at specific bin locations, we applied an innovative method of coherent amplitude-versusoffset estimation (Grion, Mazzotti and Spagnolini 1998) that uses matched filtering techniques to separate the amplitude response of the target reflections from the intersecting noise. This will be discussed in a section below.

To assess the efficiency of single-vintage processing in adjusting the data of the different vintages to be more homogeneous, we now examine the consistency of the amplitude response of our reference interface (Top Cretaceous) on the four different vintage data sets. To this end, we extract the bin gathers at the locations, A, B, C, D, on the section shown in Fig. 2, and compute the incoherent amplitude-versus-offset (Mazzotti 1991) of the Top Cretaceous reflection of the four vintages. By incoherent amplitude-versus-offset, we mean the amplitude value that, at each offset, is computed by taking the peak of the envelope amplitude in a short time window that includes the reflection. The four diagrams in Fig. 7 show the amplitude-versus-offset results for the four locations (A to D from top to bottom). The different colours of the amplitudeversus-offset curves indicate the different vintages. For location $\mathrm{C}$ we show the expected reflection coefficient computed from the borehole data of a nearby well; only in this case does the horizontal axis represent the angles of incidence on the target, and the curves are normalized to the average reflection coefficient. In the other cases (A, B and D), the horizontal axis indicates the shot-to-receiver offset, and each curve is normalized to its average amplitude. In all four locations (and in many others not shown), there is fairly good correspondence among the four different vintage amplitude responses, with agreement between major features and amplitude-versusoffset trends. The responses at location D are of particular interest. In this location, the amplitude-versus-offset relationships of the four vintages clearly do not correspond to a physically reliable amplitude-versus-offset, in fact the two maxima at around $800 \mathrm{~m}$ and $2000 \mathrm{~m}$ are due to interfering coherent noise that is evident on visual inspection of the data. However, it can be observed how this kind of noise recurs in all the vintages at approximately the same offset ranges, the exception being the 1992 data (red curve) that fall some $250 \mathrm{~m}$ further south. This suggests that this kind of coherent noise due to diffraction tails from shallower geological objects does not significantly affect our time-lapse amplitude analysis.

In conclusion, the preprocessing of each single vintage was effective in enhancing the signal content and in adjusting the data to be more homogeneous and thus suitable for further time-lapse analysis. In particular, the reflections from the Top Cretaceous reference layer show consistent amplitude responses in all four vintages. This positive outcome, although not guaranteeing that we can observe differences in the target reservoir in the event of such differences being too small, does give us confidence in the applied processing sequence. Furthermore, we start seeing a spatial variation of the amplitude response of the Top Cretaceous reflection that will be confirmed later by other results.

\section{TWO TYPES OF AMPLITUDE CROSS-CALIBRATION}

It is now possible to perform an amplitude cross-calibration of the four vintages, taking as reference the reflection of the Cretaceous layer. It must be remembered that this layer was chosen as the reference because it marks a strong impedance contrast and because it is located well above the reservoir, thus no changes in seismic response with time are expected. The aim of this phase is to remove residual amplitude differences due to the different characteristics of the vintages, and thus to obtain a constant seismic amplitude response for the reference horizon. Should differences be found after this cross-calibration for the Base Brent responses in the various vintages, then such differences could be ascribed to variations in the reservoir.

We used two different prestack amplitude cross-calibration approaches that we call amplitude-versus-offset crosscalibration and data amplitude cross-calibration.

\section{Amplitude-versus-offset cross-calibration}

The amplitude-versus-offset cross-calibration is carried out using the amplitude-versus-offset trends at the end of the singlevintage processing (such as those shown in Fig. 7) and does not modify the actual values of the seismic samples. A plane was fitted, in offset-bin coordinates, to the amplitude-versus-offset curves of the Top Cretaceous reflection of the 1989 reference vintage. Similarly, other planes were fitted to the 1982, 1992 


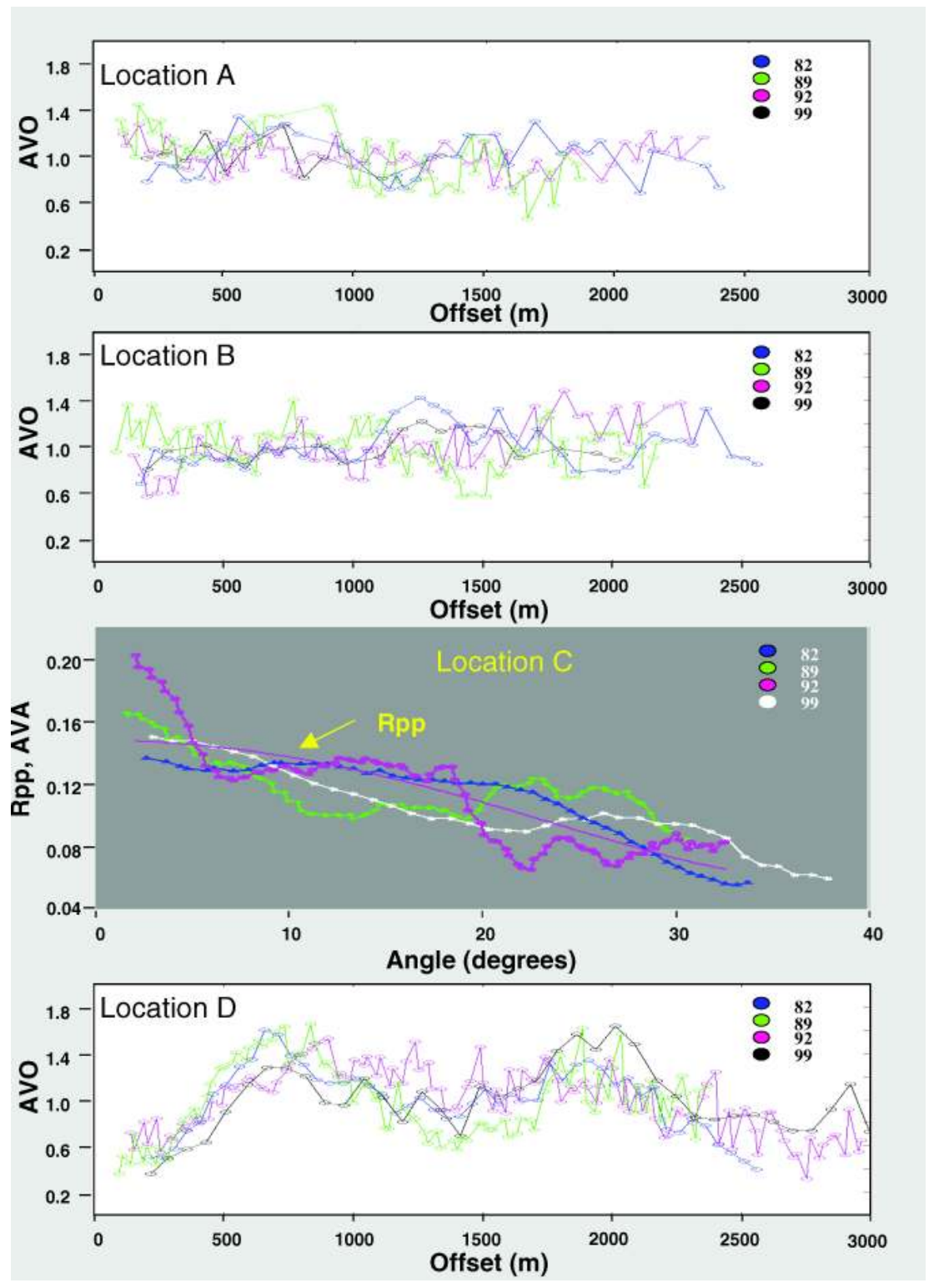

Figure 7 Incoherent amplitude-versusoffset curves (peak amplitude of the envelope) after single-vintage processing for different locations (A, B, C, D; Fig. 2). Different vintages are indicated by colour codes. Note that for location C (close to the borehole) the horizontal axis represents angles of incidence and not offset. In this case we also plotted the theoretical P-wave reflection coefficient $\left(R_{\mathrm{PP}}\right)$, computed from borehole information: the major features of the observed amplitude-versus-angle-ofincidence curves are in agreement with the $R_{\text {PP }}$ trend.

and 1999 amplitudes versus offset and versus bin, and calibration coefficients were then computed to make the 1982, 1992 and 1999 planes coincide with the reference plane of 1989. These calibration coefficients were then applied to the amplitudes versus offset and versus bin of the Base Brent reflection. Thus, this calibration is applied only to the amplitude-versusoffset curves, not to actual seismic data. The same procedure, using hyperplanes, could be applied to the full 3D data ( $x$-bin, $y$-bin and offset coordinates).

We tried the same fitting procedure making use of higherorder surfaces (second-order in offset) but the results we obtained did not differ significantly from those obtained by fitting planes.
To check the effects of the amplitude-versus-offset crosscalibration, either the cross-calibrated amplitude-versus-offset curves and the original amplitude-versus-offset curves of each bin were then fitted by Shuey parabolic curves, and intercept and gradient attributes were computed. Figures $8(a, b)$ show the amplitude-versus-offset intercepts of the Top Cretaceous reflections for the coincident profiles of 1982, 1989 and 1999, before and after the amplitude-versus-offset cross-calibration, respectively. A reasonable matching of the intercept values of the three vintages along the whole Top Cretaceous interface after the calibration was achieved. Since the amplitude-versusoffset cross-calibration was performed by matching the planes fitted to the original amplitudes and not by matching the Shuey 
Figure 8 Amplitude-versus-offset intercept at the Top Cretaceous reflection (a) before and (b) after the amplitude-versus-offset calibration. These two figures range from $x$-coordinate 5000 to $x$-coordinate 11000 on the map shown in Fig. 1 and the stack shown in Fig. 2.

Figure 9 Amplitude-versus-offset gradient at the Top Cretaceous reflection (a) before and (b) after the amplitude-versus-offset calibration. The horizontal range is the same as in Fig. 8.
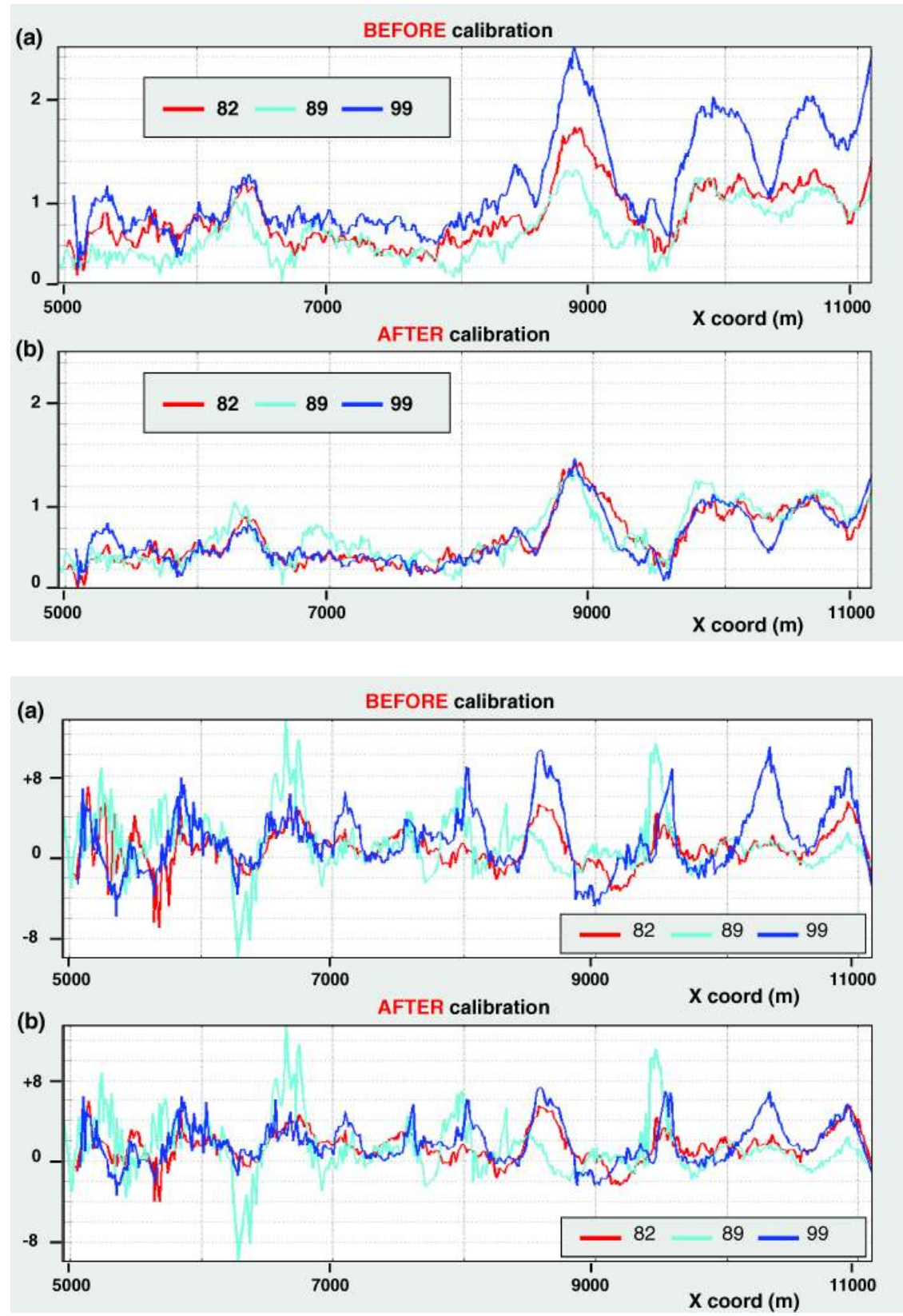

curves, it is clear that the intercepts of the three vintages may not coincide exactly. Analogous results, although more noisy, were obtained for the gradient attribute (Fig. 9).

At this stage we assume that, on average, we have removed the residual amplitude effects due to the differences in the acquisition, which our single-vintage processing was not able to correct for. We now examine the Base Brent amplitude-versusoffset curves. Any observable time-lapse variation could now be ascribed mainly to a variation in the physical properties of the reservoir. Figures $10(a, b)$ show the curves resulting from the subtraction of the Base Brent reflection intercepts 19891982 and 1999-1982, respectively. Figures 11(a,b) show the same for the gradients. Observe the differences of the intercept and of the gradient among the three spatially coincident vintage lines. Corresponding to the central portion of the bin axis (horizontal coordinates 5200-6050), a positive variation of the intercept and a negative variation of the gradient from 1982 to 1999 can be seen (Figs $10 \mathrm{~b}$ and $11 \mathrm{~b}$ ). However, for the 1982-1989 vintages (Figs 10a and 11a), the intercept and the gradient attributes do not show differences as significant 


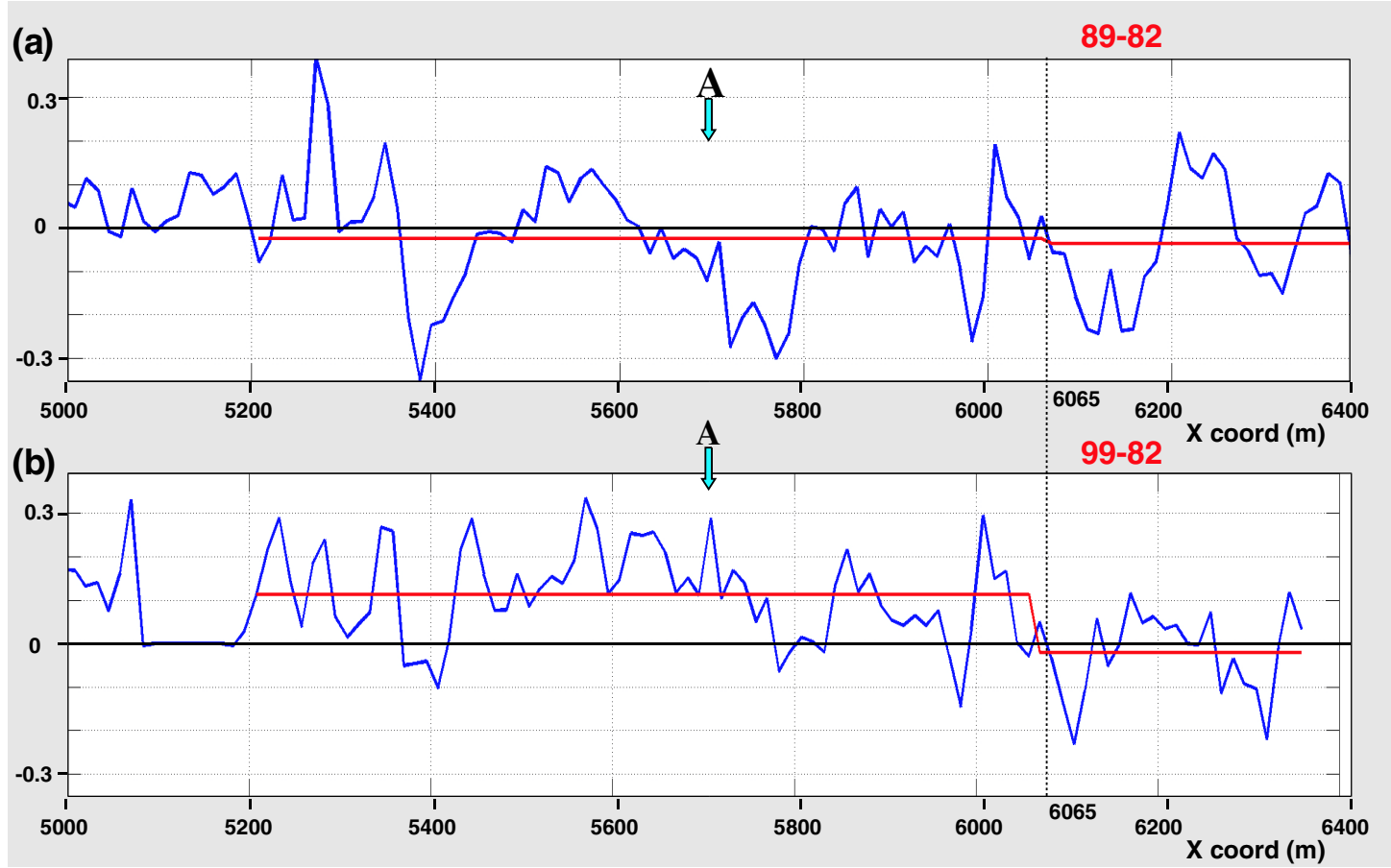

Figure 10 Differences in the intercept attribute after amplitude-versus-offset calibration along the Brent reflections: (a) 1989-1982 vintages; (b) 1999-1982 vintages. Position A corresponding to the apical part of the Brent reservoir is indicated. The red curves represent the mean values of the attributes in the intervals between $x$-coordinates 5200-6065 and $x$-coordinates 6065-6400. Noticeable variations can be observed only for the 1999-1982 vintages (b), in the $x$-interval 5200-6065.

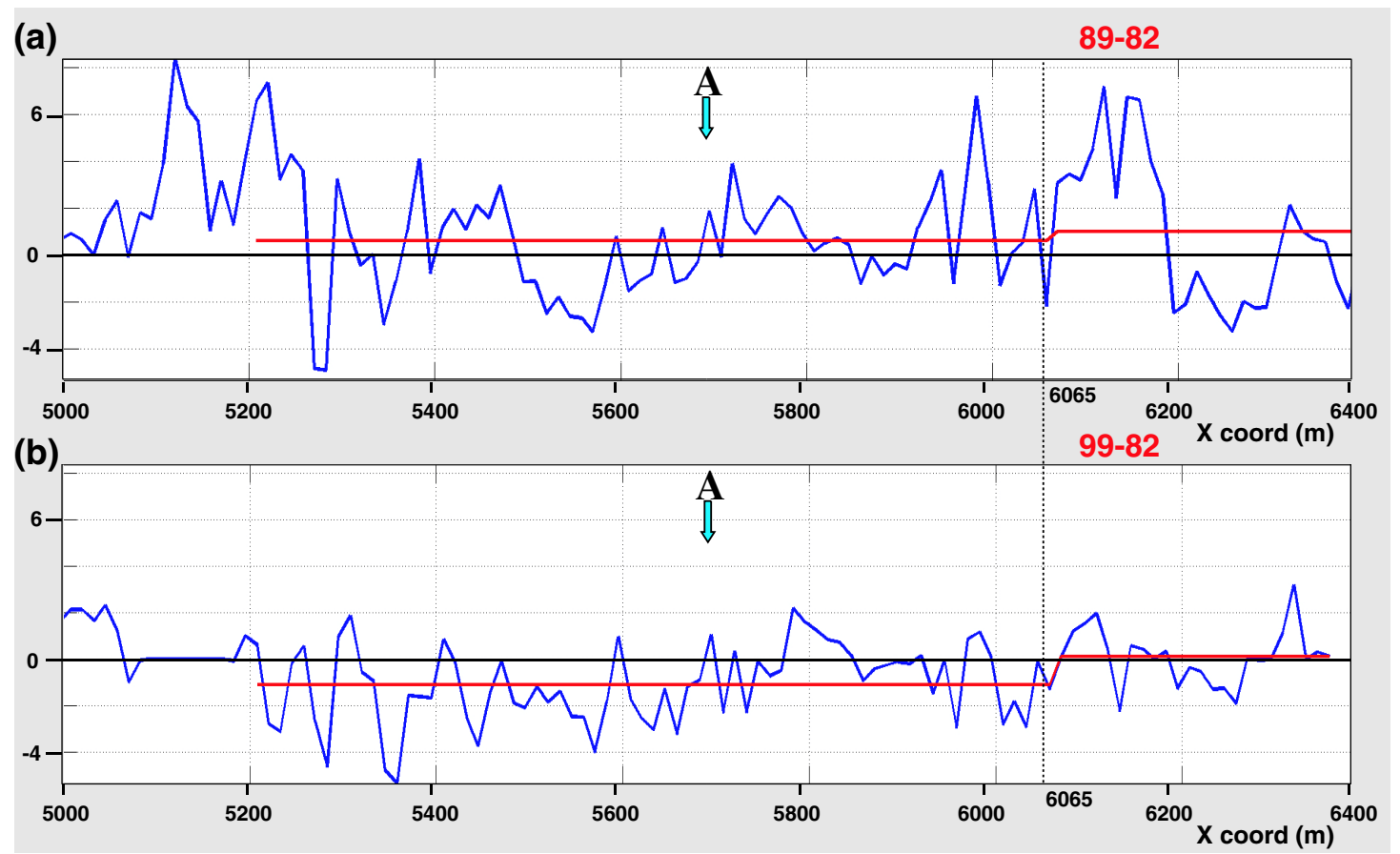

Figure 11 Differences in the gradient attribute after amplitude-versus-offset calibration along the Brent reflections: (a) 1989-1982 vintages; (b) 1999-1982 vintages. The red curves represent the mean values of the attributes as in Fig. 10. 
Figure $12 \mathrm{Rms}$ amplitudes versus vintage and channel after single-vintage processing, evaluated on bin gather traces along a time window including the reflections from the Cretaceous reference layer (see pink dots in distribution for all the vintages is shown in the histogram. Fig. 2). The overall cumulative amplitude

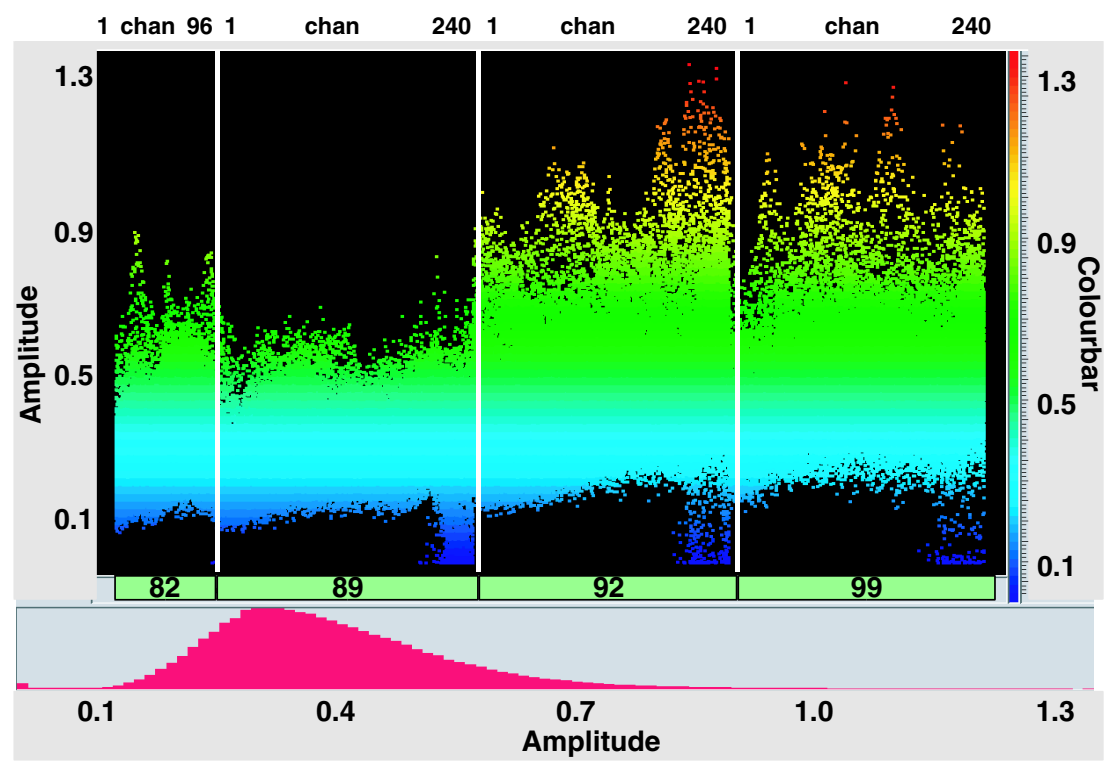

Figure $13 \mathrm{Rms}$ amplitudes versus vintage and channel as in Fig. 12, but after data amplitude cross-calibration. Note a more balanced amplitude distribution among the different vintages. Traces with anomalous amplitudes, related to the presence of noise in the data, appear at the tails of the histogram and can be removed.

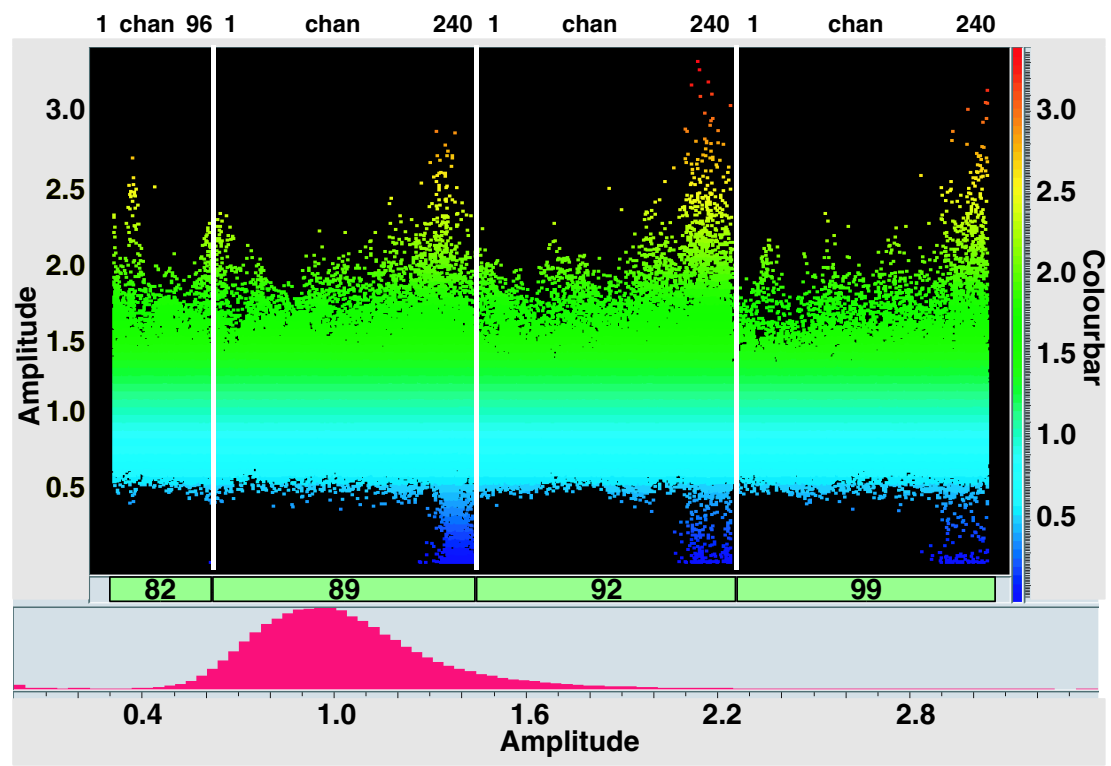

as those from 1982 to 1999 . Also, their polarity is different. The red segments indicate the average value of the plotted attribute in the portions of the Brent reflector where variations were also observed with the data amplitude cross-calibration approach described in the next section.

Data amplitude cross-calibration

In contrast to the previous method, this method modifies the amplitudes of the seismic data volume. The use of the Top Cretaceous reflections as reference data for the calibration is analogous to the previous method.
The rms amplitudes of the Top Cretaceous reflections, within a time gate of $250 \mathrm{~ms}$ that does not include the Brent reflections, were computed on the bin gathers. The pink dots in Fig. 2 indicate the corresponding time window on the stack section. In Fig. 12, the rms trace amplitudes, in colour code, are plotted versus channels. Note the differences between the various vintages: higher amplitudes are associated with the 1992 and 1999 data while the 1982 and 1989 data have lower values. The observed amplitudes were then decomposed into vintage and channel terms following a surface-consistent approach (Taner and Koehler 1981). In practice, the vintage 


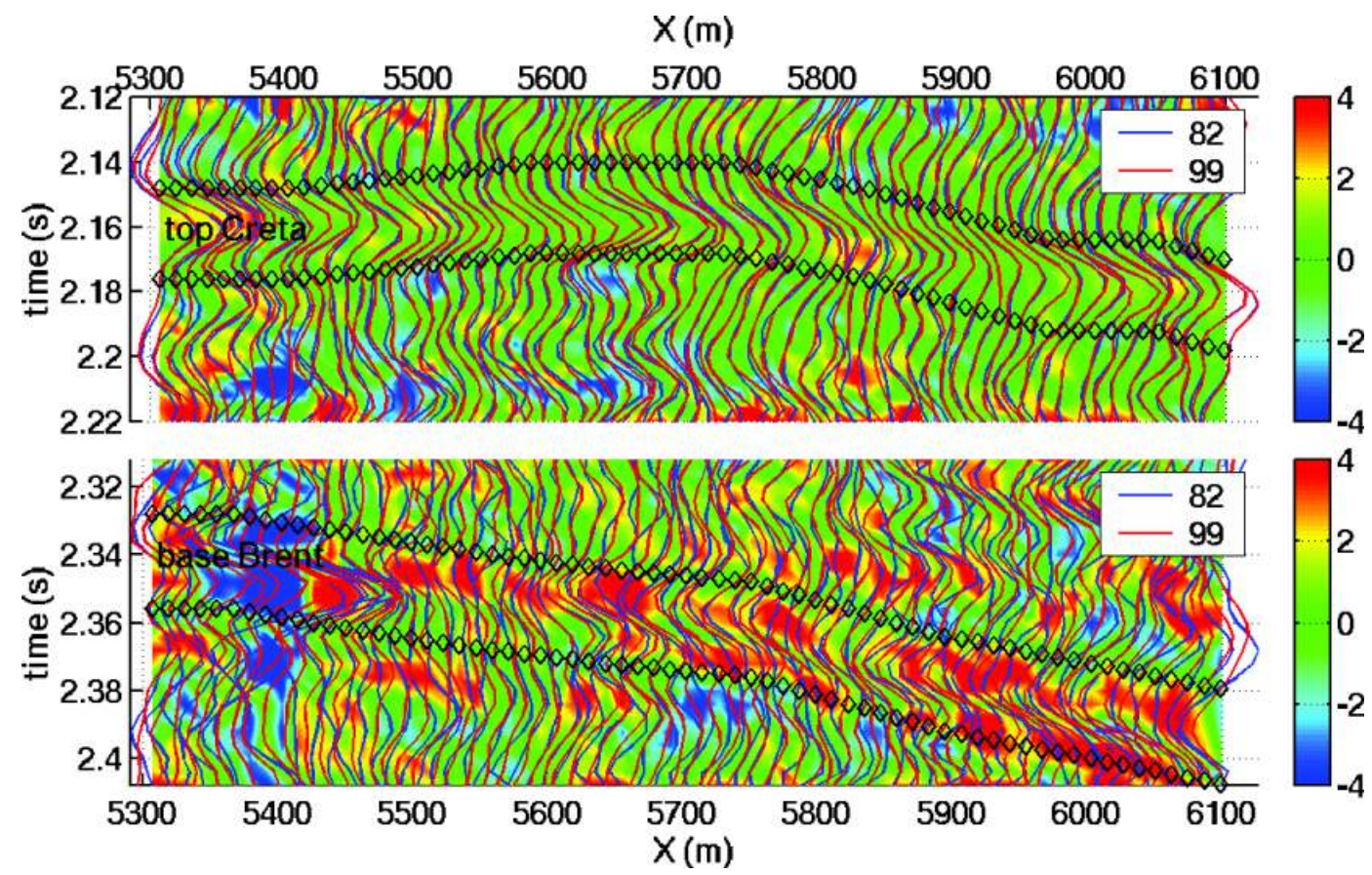

Figure 14 Close-ups of the impedance traces of the 1982 (blue) and 1999 (red) vintages computed from near-trace stack. The upper frame is centred on the Cretaceous layer and the lower frame on the Brent layer. One trace every $25 \mathrm{~m}$ is plotted. The colour scale represents the difference of the impedance moduli: 1999-1982. Impedances of the Cretaceous layer remain fairly unchanged with time. However, the 1999 impedance of the Brent layer shows a discernible and laterally continuous decrease with respect to the 1982 impedance.

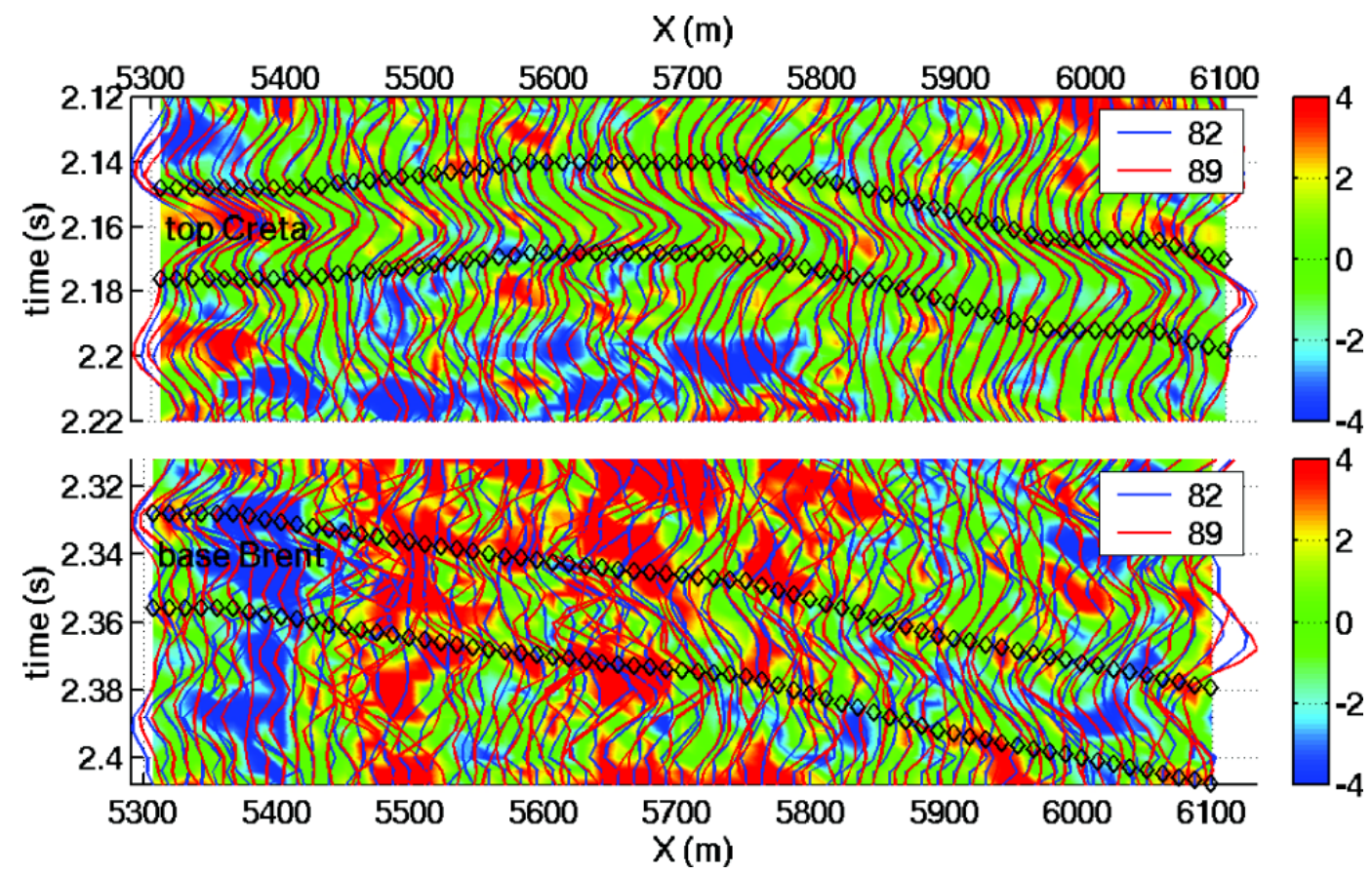

Figure 15 Close-ups of the impedance traces of the 1982 (blue) and 1989 (red) vintages. No reliable variation at the reservoir level can be identified. 


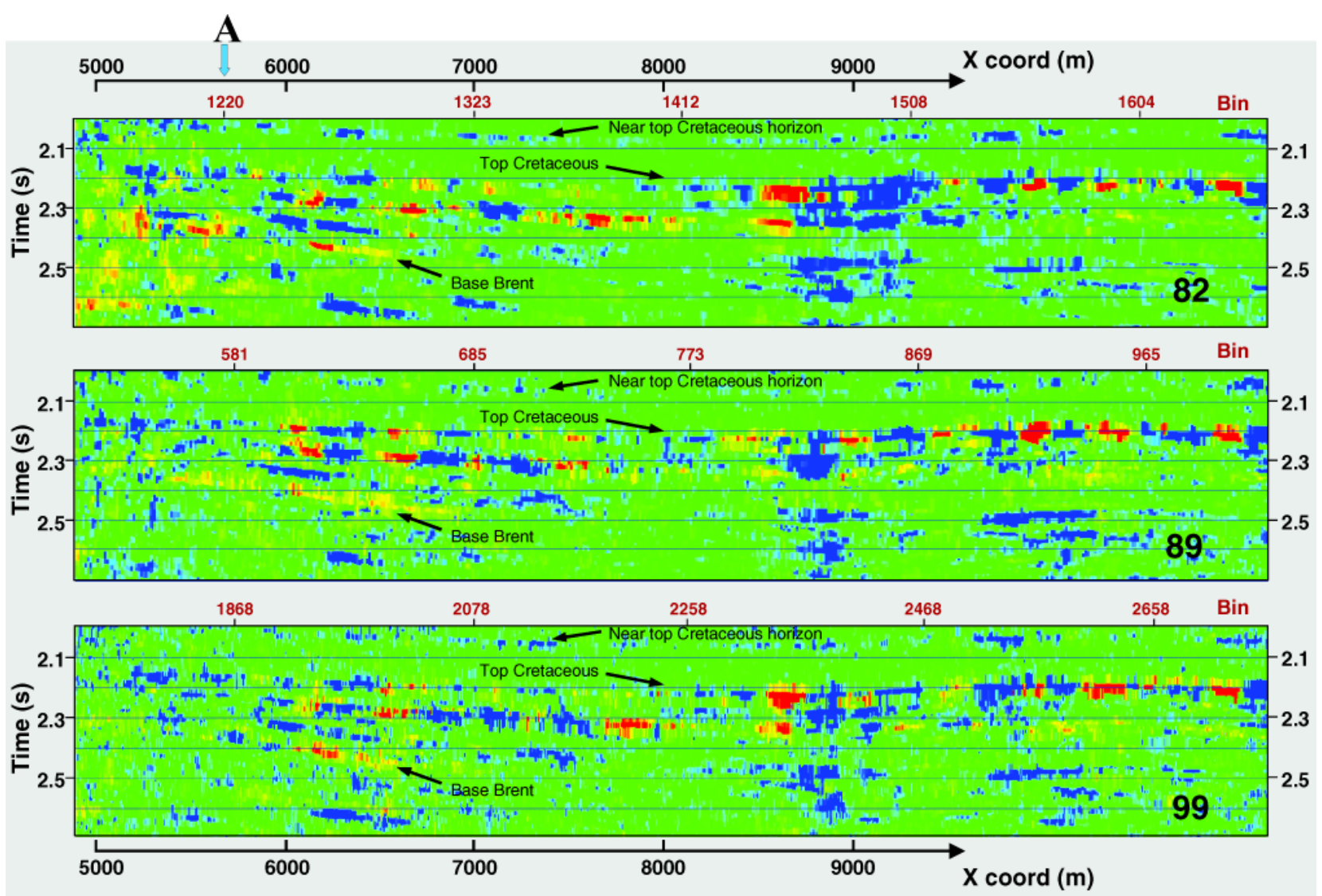

Figure 16 Intercept $\times$ gradient displays for the 1982, 1989 and 1999 data after the data amplitude cross-calibration. Note that bin numbers are different for the different vintages. For reference refer to the $x$-axis coordinates. Blue indicates a decrease in the absolute value of the amplitude with offset, red indicates an increasing trend.

terms can be thought of as the centroids of the different vintage amplitude populations shown in Fig. 12. As in the standard surface-consistent corrections, the application of the appropriate weights produces an overall amplitude balancing, shifting the centroids to a constant amplitude and equalizing the amplitudes with respect to the channels (Fig. 13). Additional diagnostic histograms (Figs 12 and 13, bottom) show the amplitude distribution before and after the application of the weights. It can be seen that such a correction produces a more normal distribution in the amplitude histograms. The anomalous amplitude values (very low or very high) visible at the tails of the histograms are, in general, associated with traces contaminated by noise and these can be easily removed from the database. The data amplitude crosscalibration consists of applying the weights computed for the Top Cretaceous reflections to all the data traces. The crosscalibration includes a band-pass filtering in a common band (9-13/45-65 Hz) and a residual static correction (maximum allowed shift $10 \mathrm{~ms}$ ) to flatten the target reflections.
At the end of the data amplitude cross-calibration, short angle (less than $10^{\circ}$ ) stacks were produced. These data underwent post-stack wavelet processing, based on the reflectivity computed from well logs. Impedance in the wavelet bandwidth was then computed, and is shown for the 1982 and 1999 data in Fig. 14 for the Top Cretaceous and for the apical part of the Brent reservoir. In the 1999 vintage, note the decrease in impedance compared with the 1982 data, corresponding to the Brent layer. Instead, the acoustic impedance of the Top Cretaceous remains more or less unchanged.

Figure 15 is analogous to Fig. 14 but with the 1989 data instead of the 1999. Again, no appreciable variation in acoustic impedance is seen for the Top Cretaceous layer. With regard to the Brent reservoir, there are no evident impedance changes between 1982 and 1989. This is probably because the 1989 data shows some noise and only one year of production has elapsed.

Thus, at this stage of the analysis, we have a second indication of a noticeable time-lapse variation, from 1982 to 


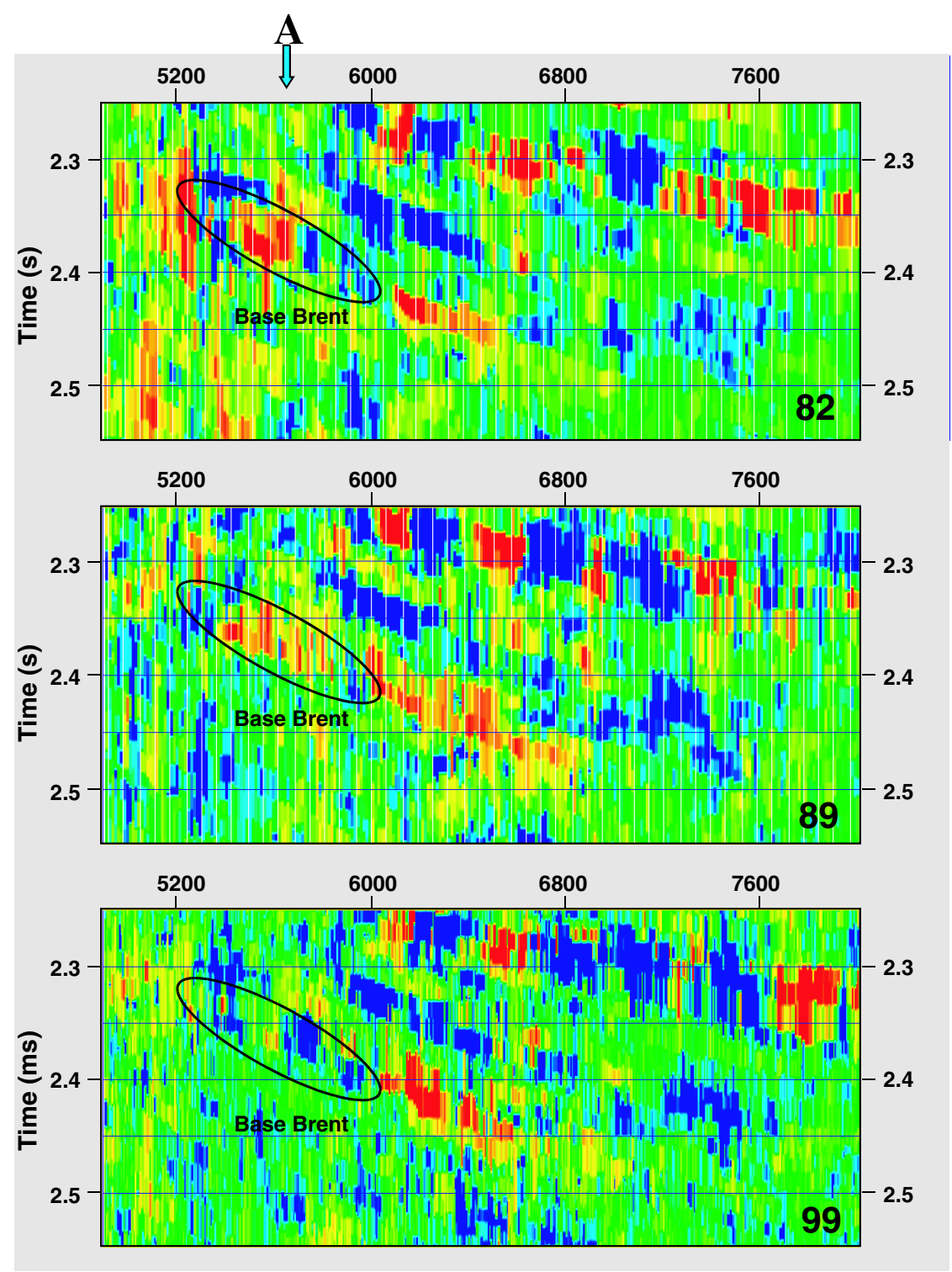

Figure 17 Close-up of Fig. 16, centred on the apical part of the Brent layer, close to location A in Fig. 2. The ellipses indicate the apical part of the Brent reservoir. Note the change of the $I \times G$ response: from red in 1982 (increase of the absolute amplitude value with offset) to blue in 1999 (decrease of the absolute amplitude value with offset).

1999, of the seismic response at near-normal incidence of the Brent layer; this is consistent with the previous outcomes of the intercept attribute obtained after the amplitude-versus-offset cross-calibration (Figs 10a,b).

We now extend the analysis to amplitudes at higher angles of incidence; as a preliminary and approximate indication of the amplitude-versus-offset responses, we compute the product of the intercept sections by the gradient section $(I \times G)$ for each vintage (Fig. 16). Since the linear approximation of the reflection coefficient is only valid for small angles of incidence, we included only reflection data with estimated angles of incidence of up to $25^{\circ}$. Although the data is still contaminated by some coherent noise, the $I \times G$ value for the horizon run- ning at about $2050 \mathrm{~ms}$ (Near Top Cretaceous) and the $I \times G$ value for the Cretaceous reflections show a reasonable match for all three vintages. The reflections from the Top Cretaceous are generally characterized by negative $I \times G$ values (blue colour). Local changes in this trend, shown as red pockets in a blue horizon, can be observed, and are fairly consistent for all vintages. However, time-lapse variations occur for the Brent reflections. These variations in the value of $I \times G$ for the three vintages are more evident in Fig. 17, which shows close-ups of the apical part of the Brent layer. Note that in 1982 and 1989, the reservoir yielded similar $I \times G$ responses (mainly red-yellow) while in 1999 , the $I \times G$ product has changed (mostly a blue response). Thus a time-lapse variation for the 
Figure 18 Coherent amplitude-versus-offset estimation of the Top Cretaceous reflections $(\mathrm{a}, \mathrm{b})$ and the Brent reflections (c, d) for 1982 data (left) and 1999 data (right). Note the invariance of the coherent amplitudeversus-offset curves for the Top Cretaceous reflections ( $a, b)$, while for the Base Brent reflections significant changes in the coherent amplitude-versus-offset curves are evident between 1982 and 1999 (c, d).
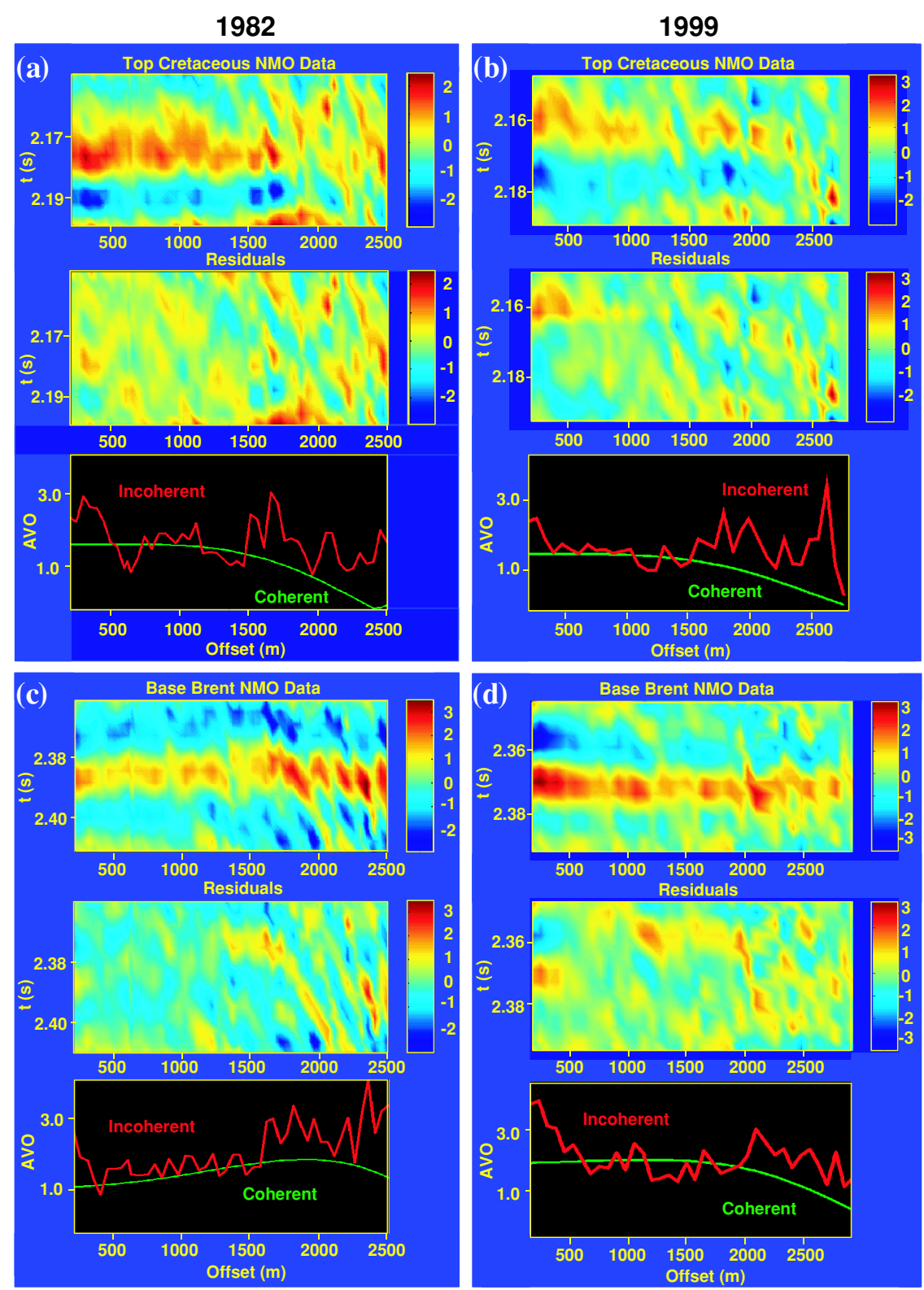

Brent layer is again visible in the $I \times G$ values computed after the data amplitude cross-calibration.

However, since $I \times G$ attributes are sensitive to noise and residual velocity error, and have various limitations, a more advanced analysis was carried out on selected bin gathers by applying a previously developed coherent amplitude-versusoffset estimation method (Grion et al. 1998). This methodology tries to determine the correct amplitude-versus-offset response of a primary wavefront in the presence of interference and random noise. Taking into account the interdependence of kinematic and amplitude factors, if velocity and amplitude- versus-offset analyses are performed sequentially, any error in velocity estimation affects the amplitude-versus-offset measures and vice versa. In order to overcome this problem, we developed an optimization technique that starts from an approximate velocity model and makes a simultaneous search for the amplitude-versus-offset and kinematic parameters that better match the observed data. The a priori knowledge of the propagating wavelet and the use of matched filtering techniques allow us to limit the distortion of the amplitude-versusoffset estimate due to random noise and interfering events, while still preserving correct amplitudes. By examining the 
residuals, i.e. the error between observed and modelled data, we could evaluate the reliability of the amplitude-versus-offset estimates. Since our objective was to estimate the optimum model for primary target reflections, the residuals should essentially contain coherent and random noise: in our specific data case, any strong contamination of the primary reflections by intersecting coherent noise will be evident. In practice, a good estimation of the wavelet and a good starting point for the traveltime description (i.e. a good velocity analysis) are needed to avoid local minima in the optimization. In our case, the wavelets of the target reflections were estimated by either singular value decomposition or wavelet processing. The results shown here were obtained using wavelets from singular value decomposition.

Figure 18 shows the results of the analysis on two bins at position A in Fig. 2: bin 1219 for the 1982 data and bin 1872 for the 1999 data. Figures $18(\mathrm{a}, \mathrm{b})$ show the results for the Top Cretaceous, Figs $18(\mathrm{c}, \mathrm{d})$ show those for the Base Brent, for 1982 and 1999. Each figure contains close-ups of the normalmoveout-corrected event, the residuals (difference between the data and the final estimated model) and the coherent and incoherent amplitude-versus-offset curves. Again, as in Fig. 7, the amplitudes of the incoherent amplitude-versus-offset curves correspond to the envelope amplitude of the target reflections. Note that the time axis of the reflections is only $50 \mathrm{~ms}$, roughly the wavelet width; thus we have full blown-up pictures of the examined reflections and optimization residuals.

The coherent amplitude-versus-offset curves resulting from the optimization are clearly less contaminated by noise and by interference than the incoherent amplitude-versus-offset curves. Thus, if the appropriate true-amplitude recovery has been carried out in the preprocessing phase, they should be close (apart from a scaling constant) to the reflection coefficient trend. The reliability of the amplitude-versus-offset estimate can be evaluated from the residuals: if these show only events not included in the model (such as diffractions and noise) and the target reflection is properly removed, then the final model found by the coherent optimization correctly reproduces the target reflection and the estimated amplitude-versus-offset is reliable. On examining the residuals in Fig. 18, it can be observed that they mainly contain steeply dipping events which intersect the target reflections, and are responsible for the undulations on the incoherent amplitude-versus-offset measures. However, the target reflections from the Top Cretaceous and from the Base Brent layers are correctly removed. Thus, we conclude that the coherent amplitude-versus-offset estimates are reliable and may be further used for quantitative studies and for inversion.
We can also check for time-lapse effects. In Figs 18(a,b), the 1982 and 1999 coherent amplitude-versus-offset curves of the Top Cretaceous are quite similar, with the same zerooffset intercepts and flat/decreasing trends. In contrast, in Figs 18(c,d), the 1982 and 1999 coherent amplitude-versusoffset curves of the Brent reservoir show different trends: from 1982 to 1999 there is an increase in the zero-offset intercept, a flatter trend at the central offsets and an earlier decrease at far offsets.

These and other coherent amplitude-versus-offset estimations on adjacent bin gathers confirm the results previously obtained with either the amplitude-versus-offset crosscalibration procedure (see Figs $10 \mathrm{~b}$ and $11 \mathrm{~b}$ ) or data amplitude cross-calibration (see Figs 14 and 17).

\section{CONCLUSIONS}

We have shown that the accurate application of simple amplitude processing sequences on seismic data from the Oseberg reservoir yields useful results.

Single-vintage preprocessing, which could also easily be applied to the entire 3D data volume, was effective in attenuating the noise components and in adjusting the amplitudes of the four vintages to be consistent. Thus, subsequent amplitude cross-calibration can be thought of as residual amplitude adjustment. The evolution of the prestack amplitudes of the data of the four vintages, both during the preprocessing phase and in amplitude cross-calibration, was accurately monitored on the Top Cretaceous reflections that were taken as reference. While the processing results have been shown for four 2D lines, each extracted from a different vintage 3D survey, the final comparison and evaluation of results in terms of timelapse amplitude variations was performed on the lines from the 1982, 1989 and 1999 vintages that are spatially coincident. In this way, we avoided the risky issue of regridding the data to a common grid.

The single-vintage amplitude preprocessing, followed by the two alternative and independent approaches of amplitude cross-calibration, produced consistent results: whereas the reflections from the Top Cretaceous reference interface show a fairly constant seismic response over time from 1982 to 1989 to 1999 , the amplitudes from the apical part of the Brent reservoir exhibit noticeable variations.

The results indicate that from 1982 to 1999 there was some variation in the relevant seismic indicators up to approximately bin coordinate 6050: namely, a decrease in the acoustic impedance, an increase in the amplitude-versus-offset intercept, and a change in the amplitude-versus-offset gradient of the Base Brent reflection. 
Coherent amplitude-versus-offset estimation at selected locations close to the apical part of the Brent reservoir retrieved more reliable, noise-free, amplitude-versus-offset responses, confirming the previous results: this can be used for further quantitative studies.

The results are consistent with an extension of the gas zone during production, as shown for the 1989-1992 period by Johnstad et al. (1995). However, since fluid movement in a reservoir is inevitably three dimensional, our analysis needs to be extended to $3 \mathrm{D}$ seismic data in order to make a practical contribution to reservoir monitoring.

In addition to the time-lapse amplitude variations relating to the Brent reservoir, it should be noted that the spatial variations of the amplitude response of the Top Cretaceous interface remained constant over the 17-year period. As an example, in the intercept $\times$ gradient section (Fig. 16), these variations are shown as red pockets, indicating an increase in absolute amplitude with offset, along a blue $I \times G$ horizon. A preliminary examination of the reflections producing these anomalies failed to reveal any particular kind of noise or other artefact, such as focusing, that could cause such behaviour. Thus, such anomalies could be related to lateral variations in the petrophysical characteristics of the Cretaceous layer, or to other as yet undeciphered factors.

\section{ACKNOWLEDGEMENTS}

This work was carried out within the framework of the research project '4D Tomographic and AVO Inversion for Seismic Lithology', partly funded by the EC - Thermie Programme. We thank the Oseberg licence partners for providing the seismic data used in the project, and thank our project partners Norsk Hydro, Total and OGS for their contributions. Many thanks to Steen Petersen, Jan Pajchel, Paul Williamson, Peter Rowbotham, Iain Bush, Gael Janex, Aldo Vesnaver and Gualtiero Bohm for their many fruitful discussions. We also gratefully acknowledge two anonymous reviewers for their constructive comments and suggestions. The processing at the University of Milan was carried out by means of the ProMAX software of Landmark Graphics Co.

\section{REFERENCES}

Bush I., Janex G., Rowbotham P., Mazzotti A., Stucchi E. and Ciuffi S. 2000. AVO inversion of 4-D seismic for reservoir monitoring: An example on the Oseberg Field. EAGE/SAID Petrophysics meets Geophysics, Paris, France, 6-8 November 2000, Session A4.

De Waal J.A., Calvert R.W., Staples R.K., Hartung M. and Shell Global 4D Team 2001. Shell's drive for 4D seismic. 63rd EAGE confer- ence, Amsterdam, The Netherlands, Extended Abstracts, Session F17.

Greaves R. and Fulp T. 1987. Three dimensional seismic monitoring of an enhanced oil recovery process. Geophysics 52, 11751187.

Grion S., Mazzotti A. and Spagnolini U. 1998. Joint estimation of AVO and kinematic parameters. Geophysical Prospecting 46, $405-$ 422.

Harris P.E. and Henry B. 1998. Time-lapse processing: a North Sea case study. 68th SEG meeting, New Orleans, USA, Expanded Abstracts, Session 4D 1.1.

Hicks G.J. and Williamson P. 2002. Quantitative time-lapse AVO Initial application to the Oseberg Field. 64th EAGE conference, Florence, Italy, Extended Abstracts, Session A019.

Johnsen J.R., Rutledal H. and Nilsen D.E. 1995. Jurassic reservoirs; field examples from the Oseberg and Troll fields: Horda platform area. In: Petroleum Exploration and Exploitation in Norway (ed. S. Hanslien), pp. 199-234. NPF Special Publication 4. Norwegian Petroleum Society.

Johnstad S.E., Seymour R.H. and Smith P.J. 1995. Seismic reservoir monitoring over the Oseberg field during the period 1989-1992. First Break 13, 169-183.

Levin S.A. 1989. Surface-consistent deconvolution. Geophysics 54, 1123-1133.

Lokshtanov D. 2000. Suppression of water-layer multiples - from deconvolution to wave-equation approach. 70th SEG meeting, Calgary, Canada, Expanded Abstracts, 1981-1984.

Lumley D.E. 2001. Time-lapse seismic reservoir monitoring. Geophysics 66, 50-53.

Lumley D.E., Behrens R.A. and Wang Z. 1997. Assessing the technical risk of a 4-D seismic project. The Leading Edge 16, 12871291.

Mazzotti A. 1991. Amplitude, phase and frequency versus offset applications. Geophysical Prospecting 39, 863-886.

Mazzotti A., Stucchi E. and Ciuffi S. 2000. Time-lapse seismic amplitude studies - Preliminary results from a North Sea dataset. 62nd EAGE conference, Glasgow, Scotland, Extended Abstracts, Session X56.

Morley L. and Claerbout J. 1983. Predictive deconvolution in shot receiver space. Geophysics 48, 515-531.

Parker J., Bertelli L. and Dromgoole P. 2003. 4D seismic technology special issue. Petroleum Geoscience 9 (iv).

Rickett J.E. and Lumley D.E. 2001. Cross-equalization data processing for time-lapse seismic reservoir monitoring: a case study from the Gulf of Mexico. Geophysics 66, 1015-1025.

Rowbotham P., Williamson P., Marion D., Janex G. and Gosselin O. 2001. Quantitative use of pre-stack time-lapse seismic for production history matching. 63rd EAGE conference, Amsterdam, The Netherlands, Extended Abstracts, Session N09.

Spitz S. 2000. Model-based subtraction of multiple events in the frequency-space domain. 70th SEG meeting, Calgary, Canada, Expanded Abstracts, Session SP2.

Stucchi E., Mazzotti A. and Terenghi P. 2001. Time-lapse amplitude variations on seismic data from the Oseberg field. 63rd EAGE conference, Amsterdam, The Netherlands, Extended Abstracts, Session P668. 
Taner M.T. and Koehler F. 1981. Surface consistent corrections. Geophysics 46, 17-22.

Terenghi P. and Mazzotti A. 2002. Experiences in post-stack and prestack wavelet processing on time-lapse data. Bollettino Geofisica Teorica ed Applicata 43, 131-142.

Tura A., Hanitzsch C. and Calandra H. 1998. 3-D AVO migration/inversion of field data. The Leading Edge 17, 1578-1583.

Verschuur D.J. and Berkhout A.J. 1997. Estimation of multiple scattering by iterative inversion, Part 2: practical aspects and examples. Geophysics 62, 1596-1611.
Vesnaver A., Accaino F., Bohm G., Madrussani G., Pajchel J., Rossi G. and Dal Moro G. 2003. Time-lapse tomography. Geophysics 68, 815-823.

Vesnaver A., Janex G., Madrussani G., Mazzotti A., Pajchel J., Stucchi E. and Williamson P. 2001. Target-oriented time-lapse analysis by AVO and tomographic inversion. 71st SEG meeting, San Antonio, Texas, USA, Expanded Abstracts, Session INV 2.

Wang Z. 1997. Feasibility of time-lapse seismic reservoir monitoring: The physical basis. The Leading Edge 16, 1327-1329. 